R. J. Cohen and W. T. Sullivan, III, eds.

\title{
Radio Interference in Astronomical Observatories of China
}

\author{
B. Peng ${ }^{1}$, R. $\mathrm{Nan}^{1}$, T. Piao' ${ }^{1}$ D. Jiang ${ }^{2}$ and Y. $\mathrm{Su}^{1}$
}

National Astronomical Observatories, Chinese Academy of Sciences, Beijing 100012, China

\author{
R. G. Strom
}

NFRA and University of Amsterdam, Postbus 2, 7990 AA Dwingeloo, The Netherlands

S. Wu ${ }^{1}$, X. Zhang ${ }^{1}$, L. Zhu ${ }^{1}$ and X. Liu $^{3}$

National Astronomical Observatories, Chinese Academy of Sciences, Beijing 100012, China

\begin{abstract}
We first very briefly introduce the major radio facilities for astronomical research in China, and then report on the present interference situation at major radio observatories. Some of the radio interference problems are caused by paging services, mobile phone satellites, telemetry services for power supply, waterpower and earthquake activity, or radar systems, but some causes are unknown. In the worst case, harmful to radio astronomy, the Sesan VLBI station has not been able to do any observations at $92 \mathrm{~cm}$ due to serious radio interference problems since 1992. Still more serious interference coming from satellites can be expected in the next decade. International efforts on frequency protection should be urgently pursued if ground-based radio astronomy is to survive.
\end{abstract}

\section{Introduction}

Radio astronomy in China started with solar research about 40 years ago. At present, radio telescopes covering a wavelength range from $3 \mathrm{~mm}$ to $1.3 \mathrm{~m}$ for astronomical research are in operation at Beijing Astronomical Observatory (BAO), Purple Mountain Observatory (PMO), Shanghai Astronomical Observatory (SHAO), Yunnan Astronomical Observatory (YAO) and Urumqi Astronomical Observing Station (UAO). The Bureau of Radio Management (BRM) in the Ministry of Information Industry is currently responsible for policy-making and implementation of radio frequency allocation in China.

\footnotetext{
${ }^{1}$ Beijing Astronomical Observatory, Chinese Academy of Sciences, Beijing 100012, China

${ }^{2}$ Shanghai Astronomical Observatory, Chinese Academy of Sciences, Shanghai 200030, China

${ }^{3}$ Urumqi Astronomical Station, Chinese Academy of Sciences, Xinjiang 830011, China
} 

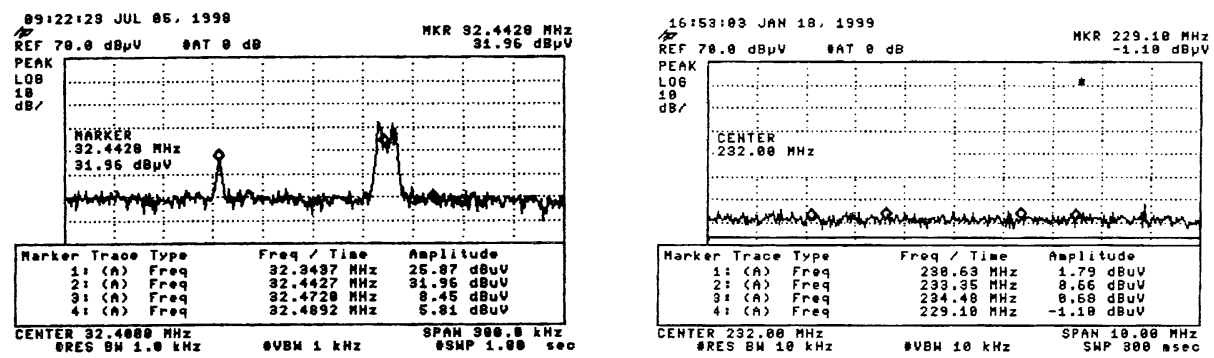

Figure 1. Left-hand plot: Interference at MSRT in July 1998 caused by a telemetry service for power supply, accompanied by mobile communication at $234 \mathrm{MHz}$ in Beijing, $\mathrm{LO}=202.00 \mathrm{MHz}$; right: Spectrum in January 1999 after the telemetry service for power supply, accompanied by mobile communication, was gone.

\section{Major Radio Astronomical Facilities}

- The Miyun Synthesis Radio Telescope (MSRT) at BAO consists of an East-West array of 28 parabolical dishes, each of $9 \mathrm{~m}$ diameter, with a longest baseline of $1164 \mathrm{~m}$ working at both 232 and $327 \mathrm{MHz}$ since 1984 .

- A $13.7 \mathrm{~m}, \mathrm{~mm}$-wave telescope run by PMO is equipped with acoustooptical and multi-channel spectrometer backends. It has been in service since 1990 at two frequency bands, 22 and $85-115 \mathrm{GHz}$.

- Two $25 \mathrm{~m}$ radio telescopes, the first built at SHAO in 1987 and the second at UAO in 1994, were put into operation, equipped with VLBA and MK3A data acquisition systems, respectively, at 5 bands $-92,18,13.6,6$ and 3.6 $\mathrm{cm}$ - and with an additional one $(1.3 \mathrm{~cm})$ at SHAO.

- Instrumentation for solar radio astronomy consists of

1. A total power radio telescope working at $2.84 \mathrm{GHz}$, and two spectrometers on 7 and $3 \mathrm{~m}$ diameter dishes receiving circular polarization with multi-channel and frequency sweeping over $1.0-2.0$, and $2.6-3.8$ $\mathrm{GHz}$, respectively, at $\mathrm{BAO}$;

2. Two total power radio telescopes at 2.7 and $9.375 \mathrm{GHz}$ at PMO;

3. A $230-300 \mathrm{MHz}$ spectrometer on a $10 \mathrm{~m}$ diameter dish, which also has continuum receivers at $1.42,2.13,2.84$ and $4.26 \mathrm{GHz}$, at YAO.

The Solar Radio Broadband Dynamic Spectrometer (SRBDS) project has been underway since 1994 and will eventually cover the frequency range $0.7-7.6 \mathrm{GHz}$.

\section{Current Interference Situation at Radio Observatories}

There are other irregular and illegal radio services within frequency bands specified for radio astronomy, situated close to observatories.

1. MSRT at BAO: two kinds of interfering signals were identified as to whether they were due to instrumental effects inside the observing room or not.

Firstly, there is some interference coming from outside the observing room, such as (a) a telemetry service for power supply accompanied by mobile 

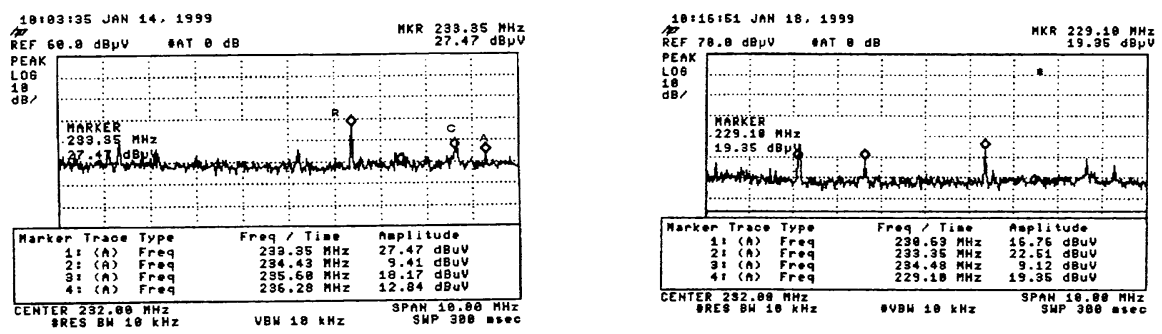

Figure 2. Interference in Jan. 1999 due to bad EMC problems generated by some subsidiary instruments inside the MSRT observing room.

phone communication at $234 \mathrm{MHz}$, and causing serious interference problems (Figure 1, left-hand plot), although the band $232 \pm 4 \mathrm{MHz}$ was said to be protected 20 years ago. After our strong and reasonable arguments with the BRM, this interference has been eliminated according to the regulations of the state management and allocation of radio frequencies, as shown in Figure 1(right-hand plot); (b) some interference around $232 \mathrm{MHz}$, caused by a military radar system and telemetry services for waterpower and earthquake activity, has been successfully removed in the same way; (c) some interference at 623.37 and $630.0 \mathrm{MHz}$ of $36.12 \mathrm{~dB} \mu \mathrm{V}$ and above 21 $\mathrm{dB} \mu \mathrm{V}$ respectively, can be well suppressed by taking defensive measures.

Secondly, some internal interference (Figure 2) is actually due to problems generated by subsidiary instrumental effects inside the observing room, such as a PC controller at 236.28 and $229.10 \mathrm{MHz}$ (signal levels of 14.18 and $15.50 \mathrm{~dB} \mu \mathrm{V})$, a GPS clock at 230.63 and $235-236 \mathrm{MHz}(15.50$ and 18.17 (maximum) $\mathrm{dB} \mu \mathrm{V})$, unshielded correlators at $233.35 \mathrm{MHz}(26 \mathrm{~dB} \mu \mathrm{V})$ and at $235-236 \mathrm{MHz}$ an average noise increase of $\sim 7.5 \mathrm{~dB} \mu \mathrm{V}$. Altogether this led to a total system noise increase of $\sim 9 \mathrm{~dB} \mu \mathrm{V}$.

2. VLBI station at SHAO: there is occasionally unknown interference in some VLBI bands. For instance, some harmful interference at L band in 1997 was recorded in Figure 3 (left-hand plot). After taking some defensive measures in 1998, such as sending the amplified RF signal directly to the observing room, and doing mixing between the RF and LO in the observing room with extra filters, the interference was clearly eliminated (Figure 3 , right-hand plot). However in the worst case, which has been very harmful to radio astronomy, the Seshan VLBI station has not been able to do any observations at $327 \mathrm{MHz}$ since 1992 due to serious radio interference mainly produced by paging transmitters.

3. VLBI station at UAO: Some interference of unknown origin shows up occasionally in some directions at $327 \mathrm{MHz}$. Recent interference monitoring in March 1999 demonstrates that there is no problem at other wavebands.

4. $13.7 \mathrm{~m}$ telescope at PMO: there has been no harmful interference.

5. Solar radio telescopes: there are several wide-band paging services around $150 \mathrm{MHz}$ in Kunming city, leading to interference problems at YAO. In addition, we have built wideband solar radio spectrographs, covering most 

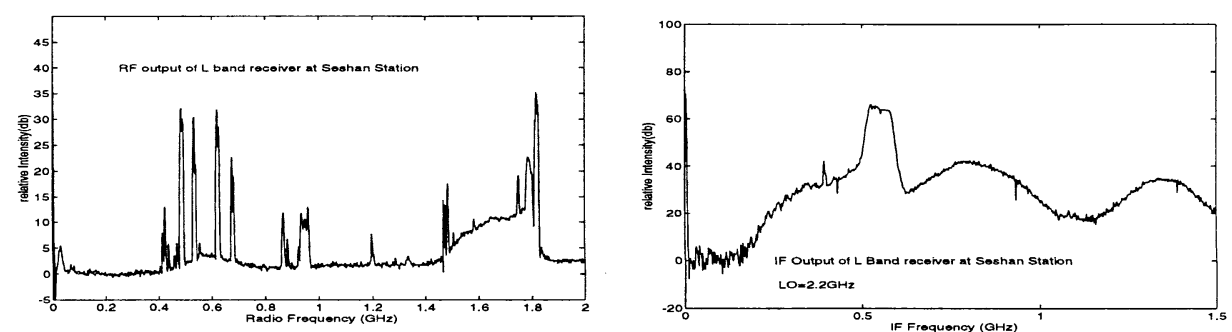

Figure 3. Left-hand plot: Radio interference at $0-2 \mathrm{GHz}$ in 1997 at SHAO. The high continuous spectrum around $1.67 \mathrm{GHz}$ is due to the operation of the preamplifier; right: Spectrum on Feb. 6, 1999 was plotted after taking some defensive measures $(\mathrm{LO}=2.2 \mathrm{GHz})$. The peak is the passband at $\mathrm{L}$ band for VLBI observing.

frequencies from 1 to $12 \mathrm{GHz}$. It is not reasonable to ask for protection for all of these from BRM.

6. Interference from Space: Satellite TV programme broadcasts cover the whole territory almost 24 hours a day. The Communication and Broadcasting Satellite Company of China was established in 1996. New groundbased stations for satellite communications have been built in Beijing and Shanghai, operating in the frequency range of $1610-1626.5 \mathrm{MHz}$. Iridium satellites have been and will continue to be launched regularly in China for the Motorola Company. More serious interference coming from space is expected in the next decade, especially in one of the most important radio astronomy bands - the $\mathrm{OH}$ band close to $1.62 \mathrm{GHz}$.

\section{Summary of Frequency Protection Efforts}

A State Standard, "Protection Criterion for the Radio Astronomy Service", has been submitted to be one of the laws for radio management in China.

Some efforts have also been made by the Chinese Astronomical Society, such as making proposals for designating a radio quiet zone in Guizhou province for a potential site of a Five-hundred-meter Aperture Spherical Telescope (FAST); appealing to the public, news media and concerned institutions to improve frequency protection for radio astronomy use; organizing national meetings to discuss issues or measures on protecting astronomical frequencies. On the other hand, new techniques have been proposed to develop telemetry or remote control instruments via power feeders instead of radio waves.

We have maintained frequent cooperation and communication with CRAF and IUCAF, exchanging information and participating in some international conferences. Cooperation with CORF takes place occasionally in some special cases. We have actively joined discussions concerning interference from satellite systems in the OECD Megascience Forum Working Group on Radio Astronomy in the past few years. We also co-proposed and signed the radio observatory directors' "Kyoto Declaration". Finally, the Asia-Pacific Commission of Radio Astronomy Frequency is to be established to strengthen efforts and collaboration on frequency protection for radio astronomy in this region.

Acknowledgments. B. Peng thanks the MPIfR in Germany and the IAU travel grants programme for supporting his attendance at this symposium. 